\title{
Passive calibration board for alignment of VIS-NIR, SWIR and LWIR images
}

\author{
by L. St-Laurent*, M. Mikhnevich*, A. Bubel* and D. Prévost* \\ *INO, 2740, Einstein Str., Québec, Canada, Louis.St-Laurent@ino.ca
}

\begin{abstract}
This paper presents a new non-electrically powered calibration rig designed for geometric calibration and spatial alignment of cameras operating in the VIS-NIR, SWIR and LWIR wavebands. Quantitative tests and experiments have been performed to identify materials, paint, varnish and configuration offering optimal properties in all wavebands. The performances obtained with this new rig were evaluated in terms of image contrast and corner detection ability. We demonstrate that the design is suitable to be used not only indoor, but also in outdoor environment with varying illumination (night, sunny and overcast day) and weather conditions (wind, cold temperature).
\end{abstract}

\section{Introduction}

Combination of sensors sensitive to different spectral bands is useful in numerous applications: inspection, surveillance and monitoring, enhanced vision, etc. An accurate pixel-by-pixel spatial registration of multi-modal images is required, or at least very helpful, for image and data fusion algorithms.

Spatial image alignment can be achieved by planar image projection (typically affine or perspective projection) after selecting and matching corresponding features observed in the scene. This is however a fastidious task due to the usual dissimilarity of features appearance in different spectral bands. Moreover, the registration thus obtained is only valid for objects located in the 2D plane determined by the selected features. This limitation becomes very important when images contain few discriminative features, as it is often observed in LWIR images.

Calibration-based registration consists in imaging a rig with geometrically known features to estimate intrinsic and extrinsic parameters. It delivers a much accurate alignment, especially when radial and tangential distortions are significant. It also provides the capability to optimize the spatial registration for various specific target distances without having to grab images of the rig at different object distances [1].

The challenge behind multi-spectral geometric camera calibration resides in having a calibration rig that produces sufficient image contrast simultaneously in all wavebands of interest. The situation gets even more complicated when calibration must be performed outdoor, where the environmental conditions (temperature, humidity, illumination, wind, etc.) change with time. To perform geometric calibration, features of the rig must be extracted and localized in images generated by all sensors. Better is the contrast (or SNR), better is the localization of the calibration rig features, and more accurate is the spatial registration of images.

Not satisfied with existing solutions, we designed a new passive (not electrically powered) calibration rig offering optimal properties for a wide spectral range: from $400 \mathrm{~nm}$ to $12 \mu \mathrm{m}$. Quantitative tests and experiments have been performed to identify materials, paint, varnish and configuration offering optimal properties in all wavebands. The performances obtained with this new rig were evaluated in terms of image contrast and corner detection ability. We demonstrate that the design is suitable to be used not only indoor, but also in outdoor environment with varying illumination (night, sunny and overcast day) and weather conditions (wind, cold temperature).

Apart from introduction and conclusion, this paper is divided in four sections. In section 2, we present a review of state-of-the-art multispectral calibration rigs. The proposed passive calibration rig is described in section 3 and a brief description of the improved features extraction algorithm is given at section 4 . Finally, some examples of calibration images in various environments are presented at section 5.

\section{Related work}

In the computer vision community, calibration rigs made of black and white checkerboard pattern printed on cardboard or paper are commonly used (left image of figure 1). Such solution is effective when all cameras are sensitive to the visible spectrum, but the visual contrast generated by these rigs in thermal images is very faint. In the SWIR range, paper shows characteristic absorption bands that may also result in contrast attenuation.

Using an active calibration rig is the preferred approach to produce very contrasting features in thermal images. A few examples of such apparatus are illustrated at figure 1. Apart from requiring to be power supplied, disadvantages of such high performance rigs are that they are bulky and more costly and complex to manufacture.

A few designs have been proposed in the literature in order to create a passive calibration rig that would exhibit sufficient contrast in both LWIR and visible spectral bands. Saporano et al. [3] use a single sheet of printed checkerboard tied with ribbon on a glazed finish ceramic tile in order to retain heat (they used a $250 \mathrm{~W}$ flood lamp to warm the board). The resulting thermal contrast is however very poor and they needed to apply aggressive contrast enhancement and nonuniformity corrections to the images to be able to perform corner detection.

Ursine et al. [4] use a checkerboard pattern made of copper squares of very low emissivity above a background painted with a high emissivity black ink to calibrate a pair of color and IR cameras. They obtained interesting results for outdoor scenes, but they need to take precautions to minimize specular reflections on their extremely low emissivity (0.09) copper material. The similar calibration rigs of Peric et al. [5] and Campo et al. [6], built with aluminum foil, also suffer from 
specular reflections. Harguess et al. [7] provided an interesting study about the choice of a calibration rig and its calibration pattern, in a context of uncontrolled outdoor scenes and wide baseline between cameras. They used a commercial

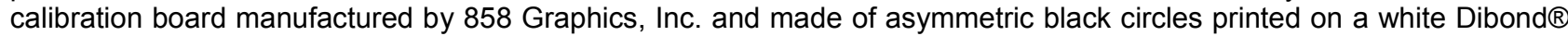
board (a composite material sandwiched between two aluminum sheets). They obtain interesting thermal contrast under sunny day, but as illustrated on left image of figure 2, their image samples reveal blurry edges caused by heat diffusion from black painted circles to the thin layer of white aluminum background.

It is also worth to notice that, to our knowledge, no work have been conducted to verify the effectiveness of a calibration rig in VIS-NIR, SWIR and LWIR wavebands simultaneously.

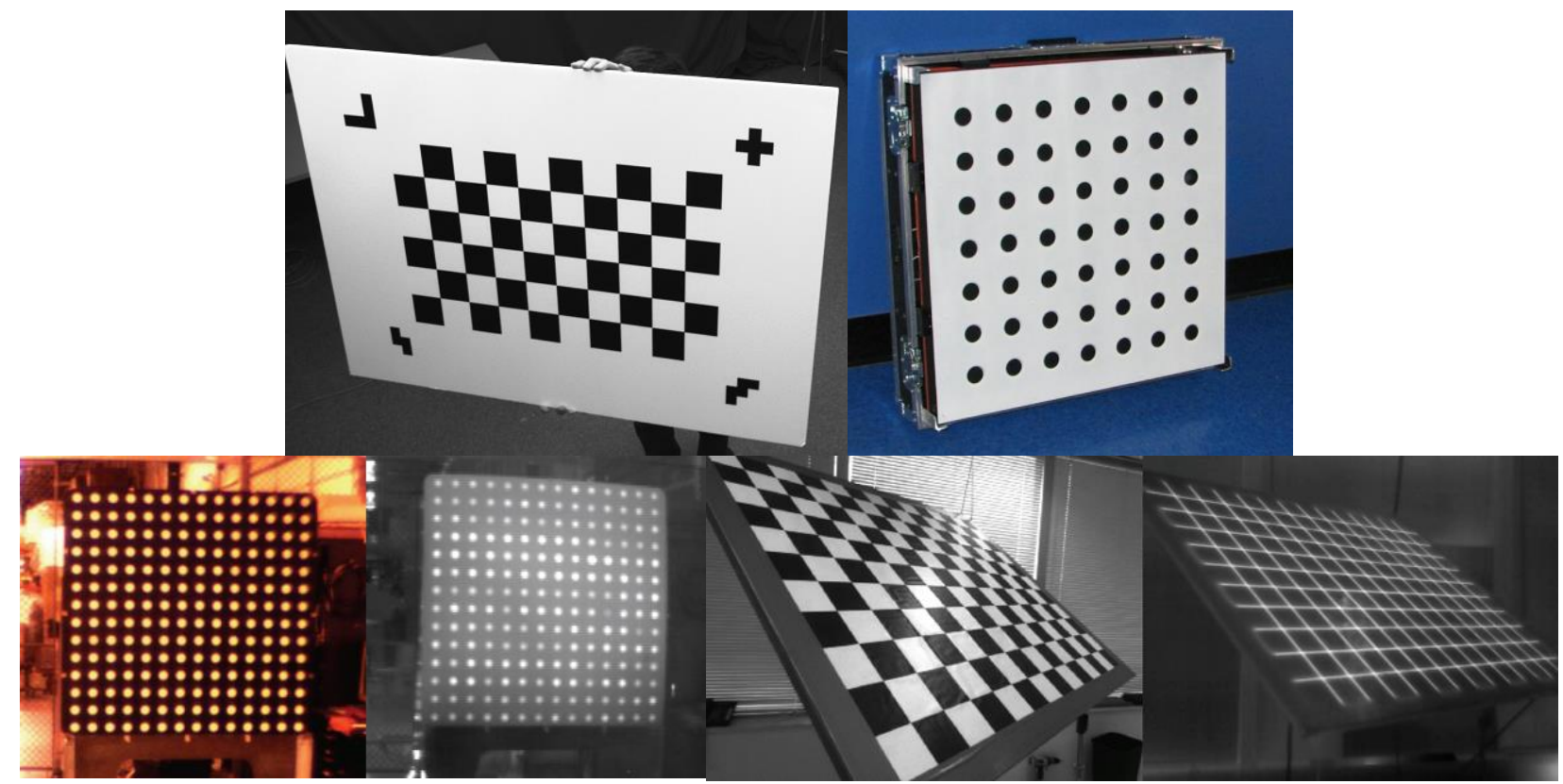

Fig. 1. Top left: cardboard rig used for calibration of color cameras of INO's Virtuo room; top right: active rig used for calibration of INO's VIRxCam platform [1]; bottom left: active rig made of black metal frame with white plastic inserts and miniature light bulb [2]; bottom right: active rig made of a grid of wires [2].
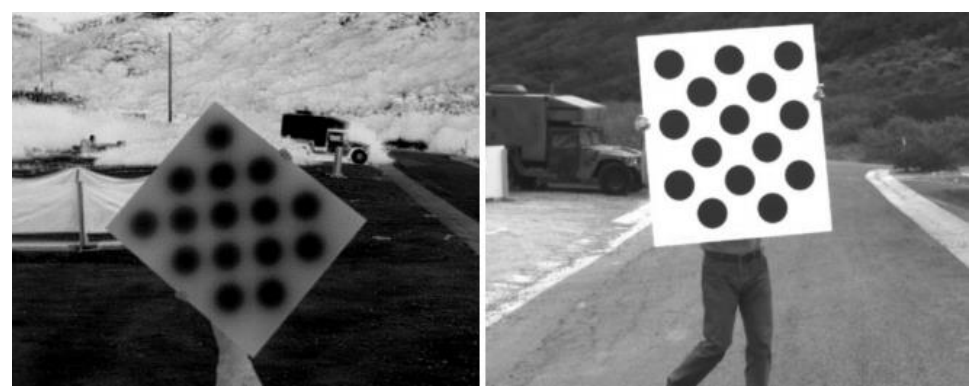

Fig. 2. Thermal (left) and visible (right) image of Dibond ${ }^{\circledR}$ asymmetric calibration rig proposed by Harguess et al.[7].

\section{Calibration board design}

Motivated by the need of registering and fusing images from sensors sensitive to VIS-NIR, SWIR and LWIR spectral bands, and not satisfied with existing solutions, we decided to design a new calibration rig. Several factors have to be considered. First of all, the chosen combination of materials of the checkerboard has to provide sufficient image contrast over the three wavebands. Secondly, the contrast in all wavebands needs to be observable and sufficiently persistent in time in all illumination and weather conditions. Finally, the rig has to be rigid, to provide accurate calibration, and lightweight, to be easily manipulated. This latter specification is critical for imaging systems with long focal length and narrow depth of field. In this situation, the calibration rig must be large enough to generate contrasting features when placed far from the imaging system. Placing the calibration rig closer to the cameras would require mechanical adjustments of focus, which would affect intrinsic calibration parameters.

Several experiments with various materials combination have been carried out. The first answer found was that applying a high emissivity black paint on a low emissivity metallic surface was among the most efficient method to generate a strong contrast in all spectral bands of interest. Moreover, the checkerboard pattern of several layers of high emissivity black ink can be by digitally printed directly onto the metallic board. Digital printing is advantageous in terms of positioning accuracy, easy repeatability and low cost manufacturing. 


\subsection{Board material}

For its low density, aluminum material is an interesting candidate. The main limitation is the strong specular reflections that completely eliminates the contrast for some viewing and illumination angles. Interestingly, we noticed that applying a sandblast finish was offering the best compromise between emissivity $(0.21)$ and reflectivity.

Aluminum composite panel, like Dibond ${ }^{\circledR}$, appeared to be an obvious choice for lightweight and rigidity. However, we observed that a slightly better contrast in the LWIR waveband was obtained with a plain aluminum plate. As illustrated on figure 3, we measured the absolute and relative contrast between the white (black painted, appearing white in thermal image) and the black areas, and found that the contrast is higher and more persistent in time with the $2.4 \mathrm{~mm}$ thick aluminum plate than with the Dibond ${ }^{\circledR}$ sample of the same thickness. Both samples were sandblasted and painted identically, then simultaneously heated the same amount of time by sweeping a halogen contractor lamp in front of them. Measurements have been done with INO's IRxCAM camera (8-12 um uncooled VOx, 640x480 pixels).
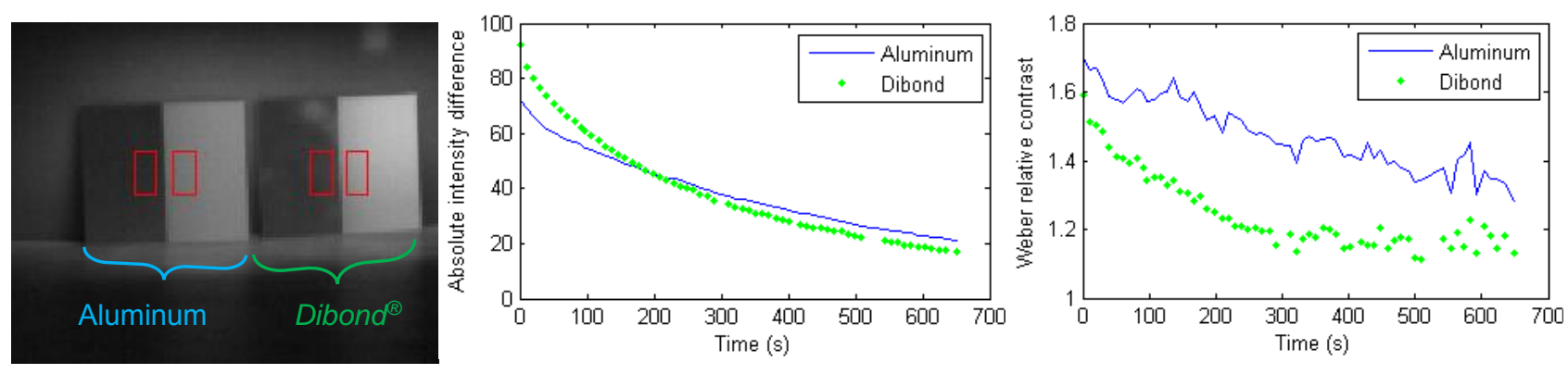

Fig. 3. Absolute (center) and relative (right) thermal contrast comparison between aluminum and Dibond ${ }^{\circledR}$ samples.

The Weber formula is used to measure the relative contrast $C$, where $I_{\max }$ is the intensity on the white sandblasted aluminium and $I_{\min }$ is the intensity on black painted sandblasted aluminum:

$$
C=\frac{I_{\max }-I_{\min }}{I_{\min }}
$$

At the beginning, the absolute contrast (intensity difference between black and white region of interest illustrated on left images of figure 3 ) of the Dibond ${ }^{\circledR}$ sample slightly exceeds the aluminum contrast. This can be explained by the fact that the exposed aluminum layer of Dibond ${ }^{\circledR}$ is thinner $(0.3 \mathrm{~mm})$, thus reaching a higher temperature. If we look at the relative contrast however, we notice that the aluminum sample exhibits a better relative contrast, even at the beginning. This indicates that the non-painted area of the Dibond ${ }^{\circledR}$ sample also increases significantly in temperature. During the dozen minutes recording time (and especially during the first 5 minutes), we can see that the contrast of the aluminum sample decreases more slowly. The aluminum layer of Dibond ${ }^{\circledR}$ being thinner, the heat dissipates faster. In outdoor environment with potential wind, the better heat retention of plain aluminum becomes an important advantage.

\subsection{Paint and varnish}

To maximize contrast and lifetime, applying matt varnish has also been considered. The contrast obtained with different printing configurations have been evaluated with INO hyperspectral system [8] in the SWIR range. Sandblasted aluminum was the substrate of all samples. Note that these measurements have not been performed in the visible waveband since SWIR and VIS cameras show the same behavior for the tested materials.

Table 1. Weber relative contrast measurements in SWIR range for different configurations.

\begin{tabular}{|c|c|c|}
\hline & Sample & Contrast \\
\hline Unpainted & thin layer black paint & 2.39 \\
\hline Unpainted & thick layer black paint & 3.74 \\
\hline Unpainted + thin layer varnish & thick layer black paint + thin layer varnish & 2.79 \\
\hline Unpainted + thick layer varnish $\mathrm{v}$ & thick layer black paint + thick layer varnish & 3.17 \\
\hline
\end{tabular}

As can been seen in table 1, it appears that printing several layers of black ink is profitable. However, the addition of varnish, although useful for the protection of the board against dirt, tends to reduce the contrast. The decrease of contrast in presence of varnish is much more amplified in the LWIR range because of its higher emissivity. This is illustrated on left image of figure 4 , where the right part of the calibration board has been recovered with varnish. Furthermore, varnish makes the calibration rig more sensitive to specular reflection. For these reasons, varnish has been discarded and the retained calibration pattern is produced only by the printing of a thick layer of black ink.

Figure 4 shows the high contrast that can be achieved with the calibration rig in the three wavebands. In this example, the acquisitions were taken at night. No heating of the board was necessary since the temperature gradient generated by getting the rig outside of an indoor room was sufficient. Note the contrast inversion in the LWIR image due to the higher emissivity of squares painted with black ink. 

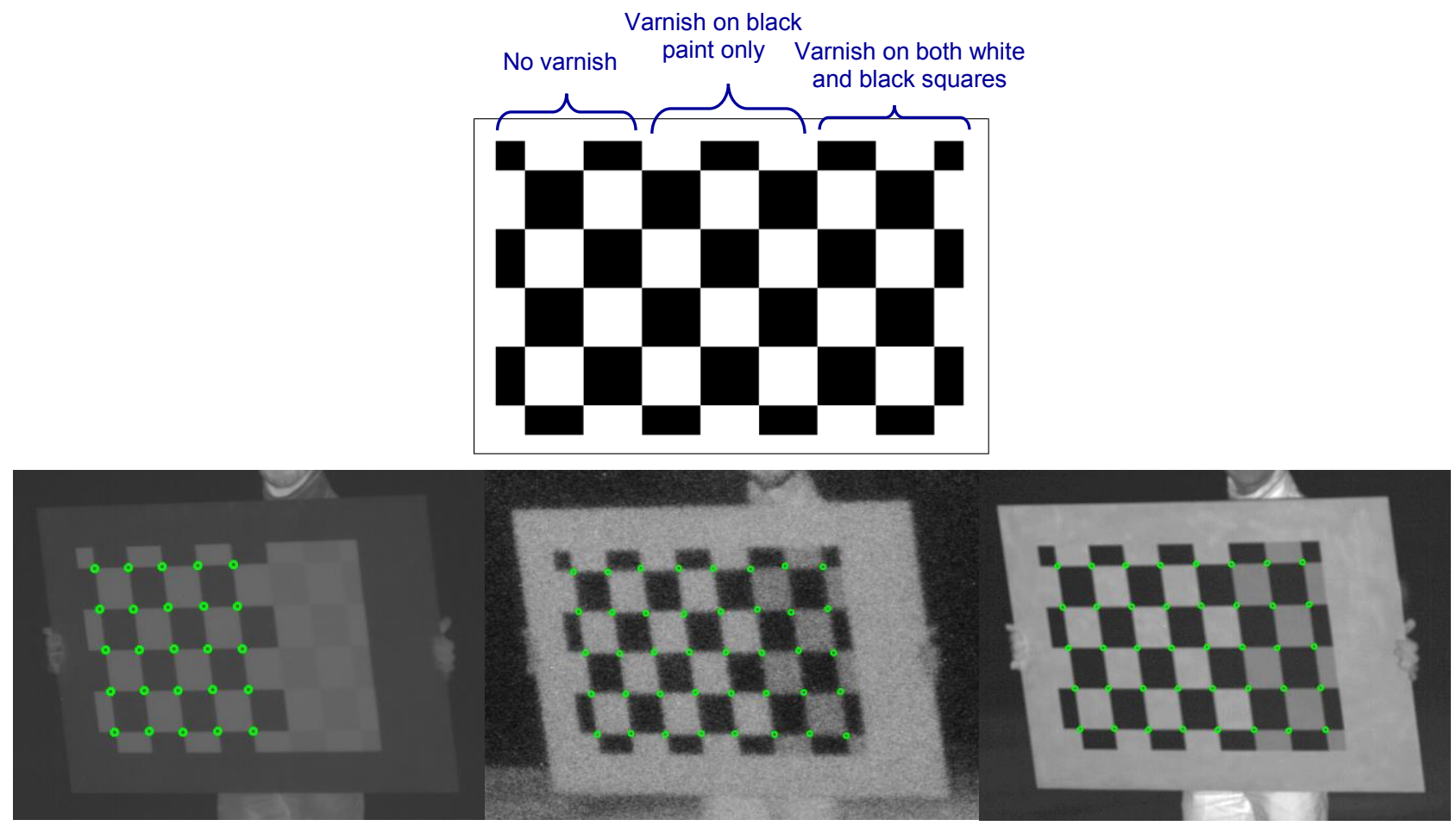

Fig. 4. Varnish test sample of the calibration rig viewed in LWIR (left), intensified visible (middle) and SWIR (right) wavebands.

\subsection{Features size}

To calibrate imaging systems with long focal length and narrow depth of field, the rig must be placed far from cameras. The checkerboard squares must thus be sufficiently large to provide contrast, even in thermal images. On the other hand, a higher number of squares provides more features and, usually, a more accurate and robust camera parameters estimation.

Based on our experiments, the final design of our calibration rig is a checkerboard made of squares of four inches wide $(101.6 \mathrm{~mm})$. A first prototype of $36 \times 24$ inches was fabricated. As illustrated at first and last rows of figure 7, this first version provides $8 \times 5$ corner features for calibration. A second rig of $40 \times 28$ inches has been fabricated. Although this second prototype was slightly larger than the first one, it only provides $7 \times 4$ corner features. However, as illustrated on center row of figure 7, this second rig is only composed of full size (4 inches wide) squares, and a 4 inches wide border is conserved all around the checkerboard. These two subtle differences make this second rig slightly more robust in presence of strong noise, and when the calibration rig is far from the cameras.

\subsection{Stiffness}

To perform accurate estimation of intrinsic and extrinsic parameters, the calibration rig must not bend when manipulated in any orientation. A thicker aluminum board would thus be suitable. However, the weight of the calibration rig rapidly becomes an issue for easily handling of a big dimensions rig. Therefore, a thin 3/32" (2.4 mm) aluminum sheet has been chosen, but to provide sufficient stiffness, the four exceeding borders (required anyway for digital printing) have been folded and bolted. Weight and stiffness are thus optimized.

\section{Target detection}

Dealing with different environment conditions (temperature, day/night illumination, etc.), with wide distance ranges (from several centimeters to tens of meters), and with multiple wavebands highly complicates target detection process. To deal with these conditions, a very robust solution for automation of calibration rig detection is required.

Our algorithm is based on the improved version of OpenCV chess board detector implemented by Rufli et al.[9]. This algorithm is fast, more robust than OpenCV chess detector and handles well high image distortions. However, it encounters problems with highly noisy images, or with the case when overall target size is less than half of a quarters of the image size.

The algorithm in [9] consists of the following steps: first, a binary image is obtained by adaptive thresholding with a constant window size estimated as $20 \%$ of the smallest image dimension. Next, the largest connected pattern of quadrangles is detected while applying morphological dilation several times. Finally, the algorithm tries to identify missing quadrangles one by one. 
The first obvious drawback of this algorithm is the constant window size for adaptive thresholding. The idea of this procedure is to use the mean of the constant size neighborhood as the threshold value for the center pixel. While this algorithm handles well images with target occupying more than half of the image, it may easily fail when the constant window size is much larger than the target size. Therefore, we run the first part of [9] with different windows sizes to estimate the average quadrangle size of the calibration chessboard, in pixels. Then, the estimated size $Q_{s}$ is used to define an optimal window size $W_{s}$ for adaptive thresholding procedure as:

$$
W_{s}=Q_{s}+\min \left(2, Q_{s} * 0.1\right) \text {. }
$$

The second problem relates to the fact that quadrangle detection is based purely on the search of a connected region of pixels in the thresholded image. This is a very efficient operation in terms of speed, but in presence of high level noise, cluttered background, or small quadrangle size, this approach often results in a high amount of false detections. To cope with this, a verification operation based on corner detector was introduced. The detected quadrangle is accepted only if at least one of its corner generates a strong responses to a corner detector. In our work, we used the recently introduced fast and efficient ChESS corner detector [10].

An additional requirement that needs to be incorporated into the target detection algorithm is robustness toward contrast inversion in thermal images. An example of such contrast inversion is presented at figure 5 where we show the evolution in time of our calibration rig after being heated. Immediately after heating the rig with a halogen contractor lamp, the black painted high emissivity squares appear white in the LWIR image. After about 16 minutes, the contrast between painted and unpainted squares located at the center of the rig completely disappears. We can even see on this frame that the contrast of the top part of the rig is inverted relatively to the contrast of the bottom part of the rig. On the frame grabbed after 26 minutes, we can notice that the contrast is now completely inverted relatively to the frame grabbed after 2 minutes. At this point, the temperature of the rig stabilized at the room temperature.

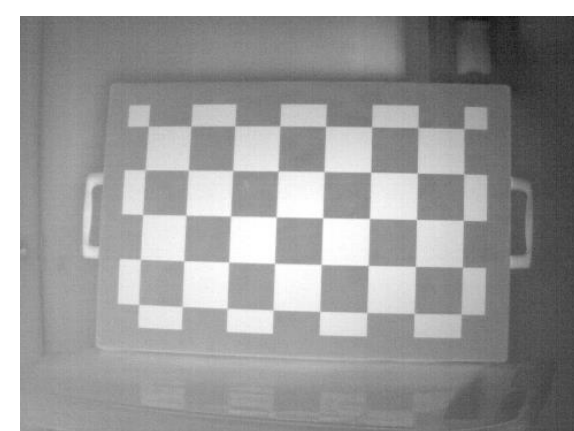

$t=2 \min$.

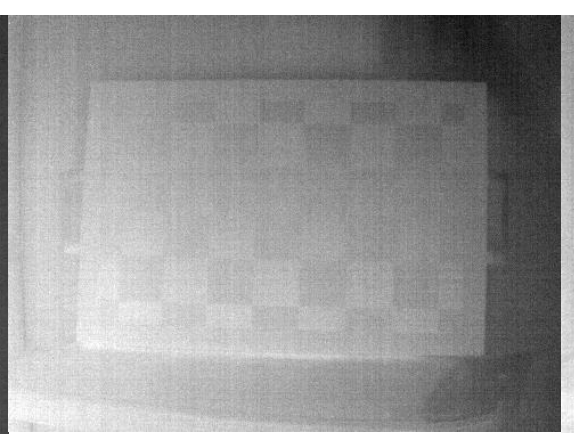

$t=16 \mathrm{~min}$.

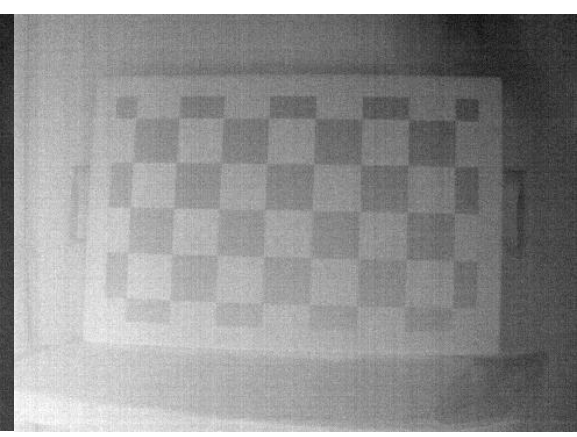

$t=26 \mathrm{~min}$.

Fig. 5. Samples of LWIR images with contrast inversion over time.

A quantitative analysis of this situation is shown on diagram of figure 6 . We can see that the absolute contrast (continuous orange line) progressively decreases until about 16 minutes, then slowly increases. During the first 11 minutes, all the 40 checkerboard corners are detected (dotted blue line). Between 15 and 20 minutes, almost no corners are detected by our algorithm. But the number of detected corners then progressively increases until about 26 minutes where all corners are successfully detected thanks to the robustness toward contrast inversion of our algorithm.

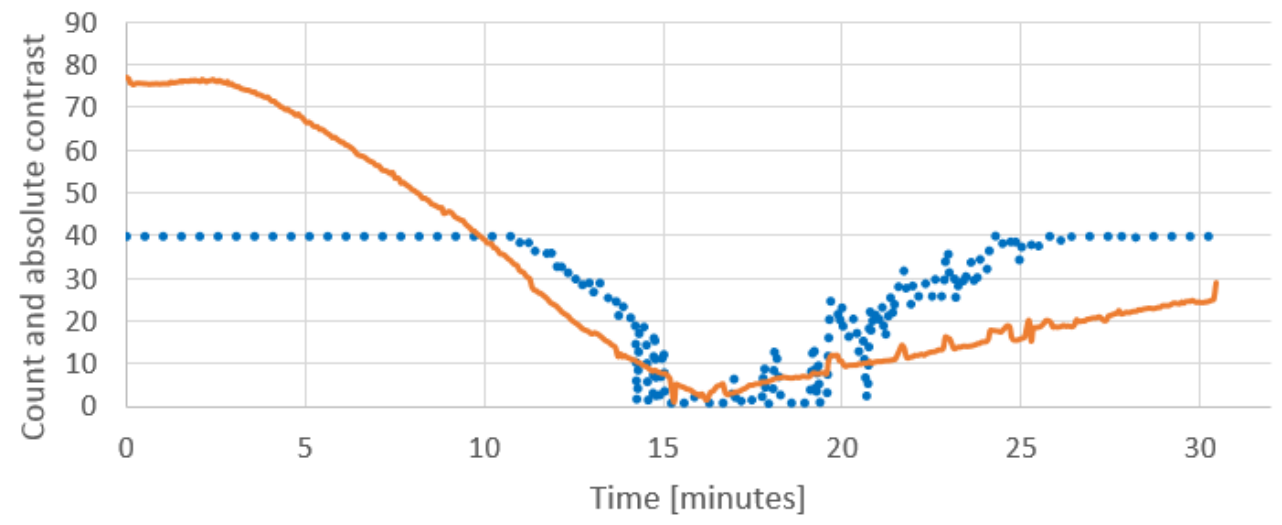

Fig. 6. Absolute contrast (continuous orange line) and number of detected corners (dotted blue curve) over time for the contrast inversion experiment illustrated at figure 5.

The example addressed at figures 5 and 6 illustrates a phenomenon of contrast inversion in time. But thermal contrast inversion can also be caused by a variation of the orientation of the rig in outdoor environment. For accurate estimation of intrinsic and extrinsic parameters, the calibration rig must be imaged at different horizontal and vertical tilt 
angles. On a sunny day in an urban scene, ground / asphalt temperature becomes significantly higher than sky temperature. When the calibration rig is oriented toward the ground, a portion of the thermal radiations coming from the ground is reflected by the low emissivity unpainted sandblasted aluminum. At the opposite, when the rig is oriented toward the sky, thermal reflections on the low emissivity unpainted sandblasted aluminum is minimal. If the temperature of the calibration rig is close to the ambient temperature, a contrast inversion might be observed between frames grabbed at up and down orientations.

\section{Performances in various environment}

Our objective is to be able to calibrate a multi-spectral imaging system under various environmental conditions (temperature, weather, time of day, etc.). We present at figure 7 some examples of corner detection results obtained indoor (top), outdoor at daytime (center), and outdoor at night time (bottom). As shown, the good contrast obtained with our calibration rig allowed us to detect almost all corners and to successfully calibrate the acquisition platform under all the tested conditions. Samples of figure 7 have been cropped manually to improve corner visibility in images. It is interesting to notice the contrast inversion applied on the LWIR image of the last row. As mentioned at the preceding paragraph, this polarity inversion is performed by our target detection algorithm in order to localize the black squares of the checkerboard when a contrast inversion affects the thermal appearance of the calibration rig.
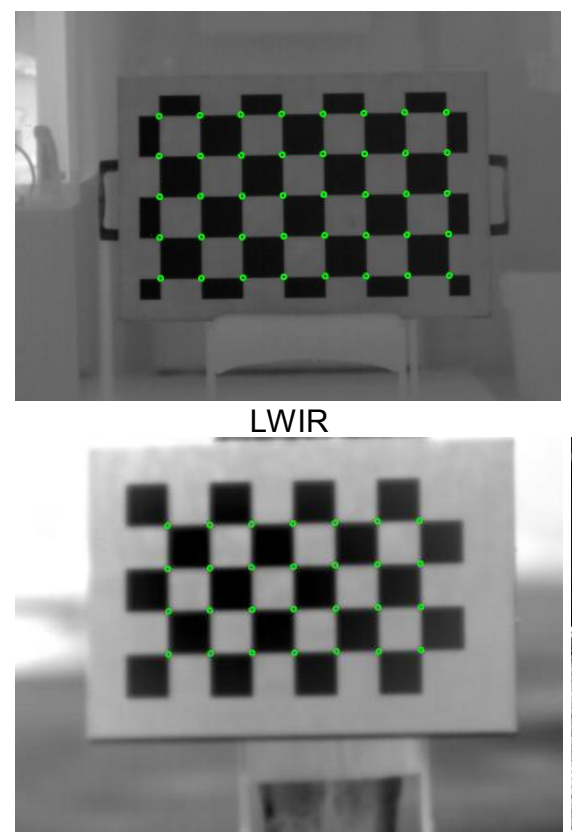

LWIR

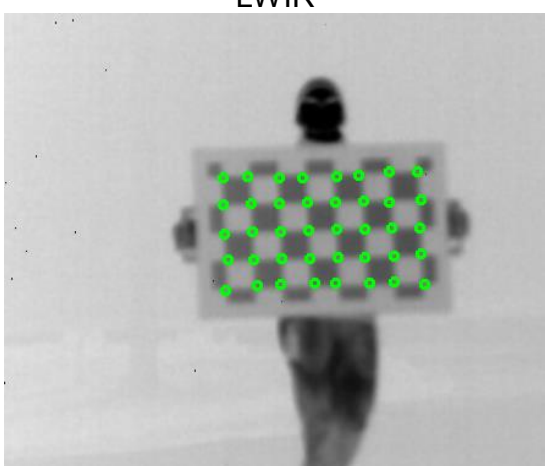

LWIR
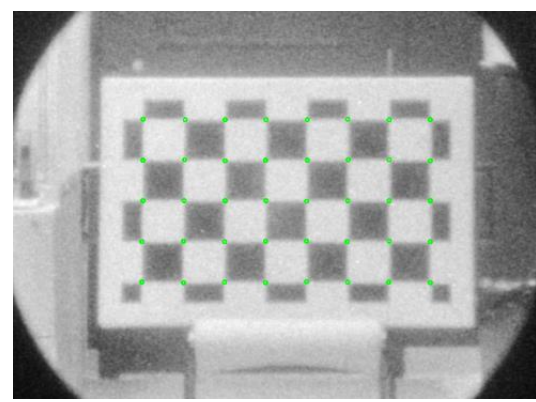

Intensified visible
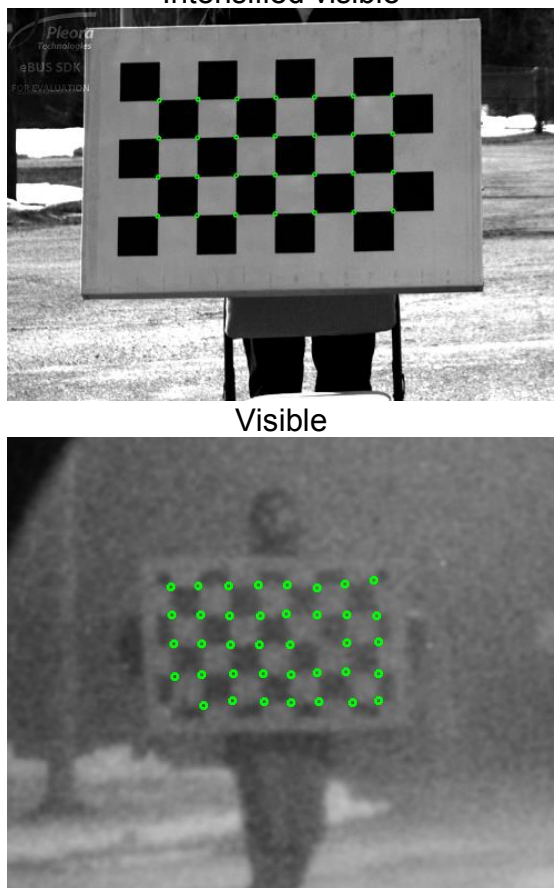

Intensified visible

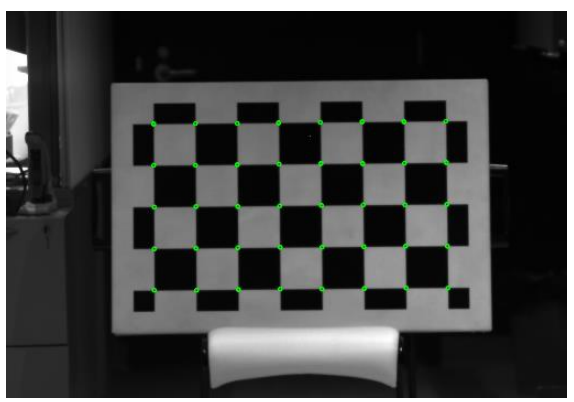

SWIR

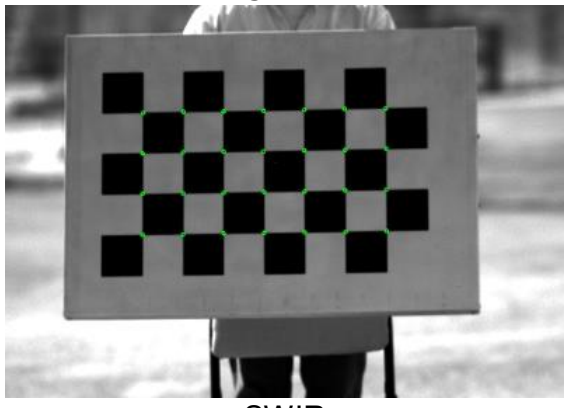

SWIR

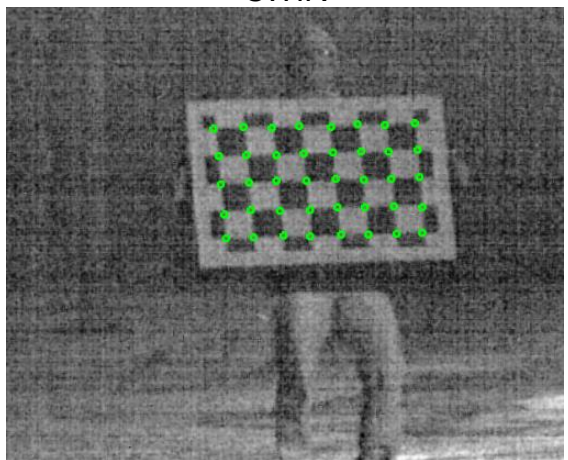

SWIR

Fig. 7. Examples of corner detection results. 


\subsection{Methodology}

A simple and versatile operation routine is desired when it comes to record the sequences of calibration images in the field (outdoor). Here are some guiding rules to optimize the checkerboard contrast depending on illumination conditions:

- Under sunlight illumination: no additional heating or illumination is required for all spectral bands.

- Overcast day: heating or cooling for thermal contrast.

- Night: heating or cooling for thermal contrast + illumination for SWIR and visible contrast.

For both heating and SWIR illumination, a standard and common contractor spotlight might be used. Alternatively, heating or cooling the rig can also be easily and very effectively performed simply by placing the rig in an environment having a different temperature. For example, letting the rig outdoor during a cold day $\left(<10^{\circ} \mathrm{C}\right)$, then bringing it back indoor for calibration provides a strong and persistent checkerboard contrast in the thermal range. Similarly, letting the rig inside a warm vehicle before to proceed to an outdoor calibration during a cold day would generates a strong thermal contrast.

The LWIR image presented at figure 4 is an example of result obtained with such methodology. It was grabbed at night and no heating of the board was necessary since the temperature gradient generated by getting the rig outside of a warm room was sufficient. Note again the contrast inversion of the LWIR checkerboard comparatively to the visible and SWIR images.

\subsection{Reprojection errors}

To estimate intrinsic and extrinsic parameters, all extracted checkerboard corners are fed to an optimization algorithm. Off-the-shelf software packages such as the OpenCV implementation of the Camera Calibration Toolbox for Matlab [11] is generally used for this task. Once the calibration parameters are known, it is possible to spatially register the images for a specific object distance. It is also possible to perform stereo or multi-views reconstruction of the scene.

In table 2, we report the reprojection errors obtained from calibrations performed indoor and outdoor. The error is expressed as the root-square-mean (RMS) difference between projected and detected target corners. RMS was measured over all views in which the calibration rig was detected. Some samples of calibration images grabbed at the different times can be visualized at figure 8 and 9 . The contrast degradation in the thermal images is clearly visible.

As predicted, the RMS reprojection error measured for the visible camera was quite stable in time. The slight fluctuations observed (like the higher 0.13 value for the first outdoor calibration) are probably caused by little variations in the poses that were selected for the calibration.

For the thermal camera, the measured RMS correspond to our expectations: with the contrast reduction, the accuracy of corner localization degrades, which leads to a reduction of the stereo reconstruction accuracy (RMS values). However, we can notice from the indoor experiment that, although the contrast of the thermal cameras was significantly decreased 10 minutes after heating (center image of figure 8 ), the reprojection error obtained with the calibration performed 10 minutes after heating $(0.18)$ is practically identical to the RMS measured at $t=0(0.20)$.

Table 2. RMS measurements in the LWIR and visible wavebands under different conditions.

\begin{tabular}{|l||c|c|c||c|c|}
\hline \multicolumn{1}{|c||}{} & \multicolumn{3}{c||}{ Indoor } & \multicolumn{2}{c|}{ Outdoor } \\
\hline Time (min) & 0 & 10 & 16 & 0 & 18 \\
\hline LWIR IRxCam RMS & 0.20 & 0.18 & 0.37 & 0.10 & 0.22 \\
\hline Visible RMS & 0.11 & 0.10 & 0.11 & 0.13 & 0.11 \\
\hline
\end{tabular}

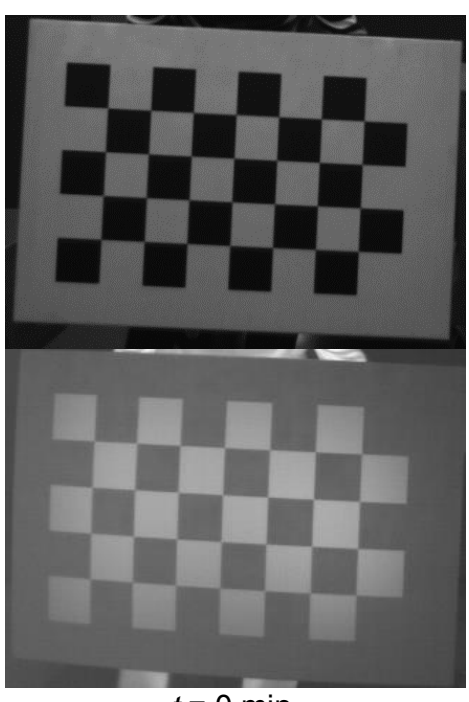

$t=0 \mathrm{~min}$.
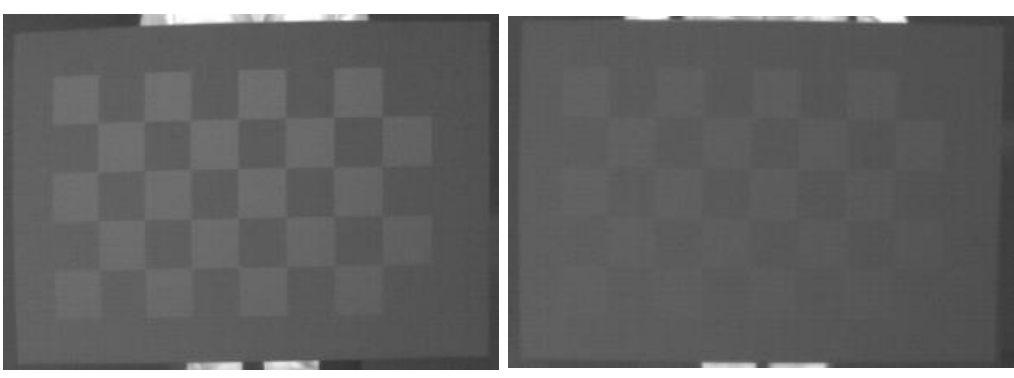

$t=10 \mathrm{~min}$

Fig. 8. Thermal and visible (top left) image samples for the indoor experiment. 


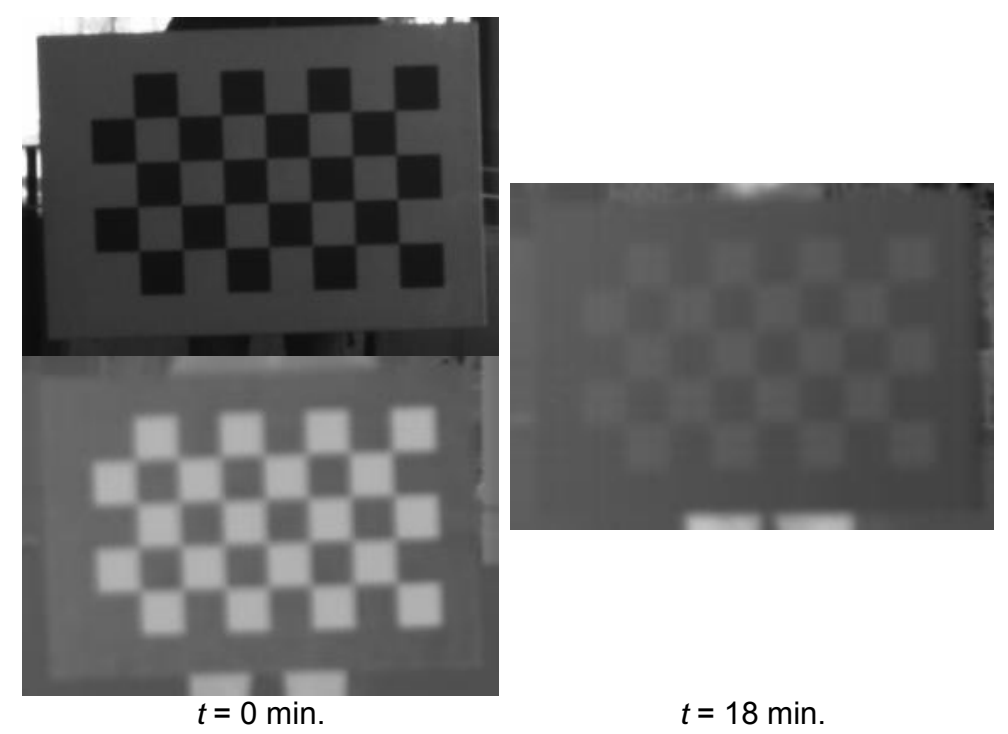

Fig. 9. Thermal and visible (top left) image samples for the outdoor experiment.

\section{Conclusion}

In this paper, we presented a passive calibration rig that produces an optimal image contrast in VIS-NIR, SWIR and LWIR wavebands simultaneously. The sandblast finish minimizes specular reflections and the plain aluminum core extends contrast persistence in time comparatively to Dibond ${ }^{\circledR}$ composite. The digitally printed checkerboard pattern is advantageous in terms of accuracy, easy repeatability and low cost manufacturing.

Two calibration rigs have been fabricated and tested on indoor and outdoor scenes. The experiments made, especially those in outdoor environments, have shown that a satisfying contrast can be obtained in varying temperature and illumination conditions. The designed rig is thus perfectly suited for accurate calibration-based registration of multi-modality imaging systems.

In the coming months and years, our calibration rigs will be used several times in all kinds of operational environments. It is planned to perform an exhaustive quantitative performance evaluation, in terms of contrast and registration accuracy, for numerous illumination and weather conditions.

\section{REFERENCES}

[1] St-Laurent L., Prévost D., Maldague X., "Fast and accurate calibration-based thermal / colour sensors registration", QIRT, 2010.

[2] Rankin et al., "Unmanned ground vehicle perception using thermal infrared cameras", SPIE Defense, Security and Sensing, International Society for Optics and Photonics, 2011.

[3] Saponaro P., Sorensen S., Rhein S., Kambhamettu C., "Improving calibration of thermal stereo camera using heated calibration board", ICIP, Quebec, CA, 2015.

[4] Ursine W., et al., "Thermal / visible autonomous stereo vision system calibration methodology for non-controlled environments", QIRT, 2012.

[5] Peric D., Likic V., Spanovic M., Sekulic R., Kocic J., "Geometric calibration of multi-sensor image fusion system with thermal infrared and low light camera", Proc. of SPIE, vol.9250, 2014.

[6] Campo F.B., Ruiz F.L., Sappa A.D., "Multimodal stereo vision system: 3D data extraction and algorithm evaluation", IEEE Journal of Selected Topics in Signal Processing, vol.6, no.5, 2012.

[7] Harguess J., Strange S., "Infrared Stereo Calibration for Unmanned Ground Vehicle Navigation", Proc. of SPIE, vol.9084, 2014.

[8] Bubel A., Renaud N., Sisto M.M., "Fusion of hyperspectral imaging and fluorescence: system and applications", Imaging and Applied Optics Congress, Arlington, VA, US, 2013.

[9] Rufli M., Scaramuzza D., Siegwart R., "Automatic detection of checkerboards on blurred and distorted images", IEEE/RSJ International Conference on Intelligent Robots and Systems, 2008.

[10] Bennett S., Lasenby J., "ChESS - Quick and robust detection of chess-board features", Computer Vision and Image Understanding, 2014.

[11] Bouguet J.Y., "Camera Calibration Toolbox for Matlab", California Institute of Technology, Pasadena, CA, US online at http://www.vision.caltech.edu/bouguetj/calib doc/index.html. 